\title{
Comparison of Cross-Sectional and Daily Reports in Studying the Relationship Between Depression and Use of Alcohol in Response to Stress in College Students
}

\author{
Heather A. Flynn
}

\begin{abstract}
Background: Alcohol use in response to stress in college students may be affected by the presence of symptoms of depression. However, this is a challenging issue to study due to the various methodologies used as well as the possible effect of depressed mood on the accuracy of self-report. This study focused on methodological issues as possible sources of equivocal findings regarding the relationship between depressed mood and alcohol use in response to stress in a college student population. Findings may differ when these variables are examined cross-sectionally versus longitudinally.

Methods: Depressed mood and alcohol coping were assessed both cross-sectionally and repeatedly over time in 125 college students. Participants were assessed at baseline using a diagnostic self-report measure of depression as well as a measure of typical coping style. In addition, daily measures of stress, symptoms of depression, and coping were completed for 45 consecutive days.

Results: Different relationships between depressed mood and alcohol coping were found when depressed individuals were analyzed separately from those who were not depressed. Although a significant correlation between daily use of alcohol coping and daily depressed mood was found, there were no differences between depressed and nondepressed participants (as assessed at baseline) on daily alcohol coping.

Conclusions: These findings have implications for research design as well as clinical assessment regarding the relationships between mood and use of alcohol for coping; the findings suggest that cross-sectional measures of mood and alcohol use may obscure differences as assessed repeatedly over time. In addition, these findings support the utility of frequent assessment of depressive symptoms when implementing or evaluating programs that target coping skills in college students.
\end{abstract}

Key Words: Alcohol Coping, Depression, Daily Data, Depressed Mood, Methodology.

$\mathbf{T}_{\mathrm{a} a \mathrm{~s}}^{\mathrm{T}}$ HIS STUDY FOCUSES on self-reported depression and daily depressed mood as related to use of alcohol to cope with stress in a college student population. Reports of dispositional alcohol coping collected at a single time point (cross-sectional reports) and daily reports of situational coping were also compared. The relatively high prevalence of dangerous alcohol use (Johnston et al., 1997) and risk for depressive symptomatology among college students (Stader and Hokanson, 1998) highlight the importance of understanding the relationships between these variables in this population. In particular, programs aimed at reducing harm and preventing relapse that incorporate coping skills education may be improved by knowledge of how the relationships among depressed mood, depression, and alco-

From the University of Michigan Medical School, Department of Psychiatry, Ann Arbor, Michigan.

Received for publication December 21, 1998; accepted October 25, 1999

Reprint requests: Heather A. Flynn, Ph.D., University of Michigan Alcohol Research Center, Department of Psychiatry, University of Michigan Medical School, 400 E. Eisenhower Pkwy, Building 2, Suite A, Ann Arbor, MI 48108-3318; E-mail: hflynn@umich.edu

Copyright 102000 by the Research Society on Alcoholism. hol use may differ depending on the timing and method of assessment.

The relationships among alcohol use, alcohol problems, and depression have received much attention in the literature (Brown and Schuckit, 1988; Turnbull and Gomberg, 1988). However, these relationships remain far from conclusive. Comparing research studies in this area is difficult because researchers have used varied populations, settings, research designs, and diagnostic criteria, including measures. For example, the relationship between depression and use of alcohol likely differs depending on whether depression is measured and manifested as depressed mood or as a diagnosed depressive disorder. Hesselbrock and Hesselbrock (1993) argued that research findings which address comorbidity of alcohol disorders with psychiatric disorders are significantly influenced by the definition and measurement of depression that are employed. In addition, these and other studies in this area typically employ a cross-sectional design. Cross-sectional or two-wave panel designs may obscure factors that determine the nature of the relationship between alcohol use and depressive symptoms. Measures of dynamic variables such as alcohol use and mood obtained at one point in time may differ from 
results obtained from repeated measurements of these variables. In addition, depressed mood at the time of assessment may influence perception and consequently reporting of alcohol use.

The nature of the relationships among alcohol use, stress, and depression, although well studied, remains inconclusive. It has been speculated, for example, that alcohol may be used in response to stress, which may result in depressed mood (Stone et al., 1985), and that alcohol may be used in response to depressed mood as a form of "selfmedication" (Hesselbrock and Hesselbrock, 1993). Studies with college student populations have found evidence of alcohol use in response to stress. In a study examining "stress-motivated" drinking in young adults, Perkins (1999) found that more than $50 \%$ of undergraduates assessed reported stress-motivated reasons for drinking. Students who use alcohol in response to stress may expect alcohol to reduce tension (Hittner, 1995) or may drink to relieve negative affective states (Weinberger and Bartholomew, 1996). The relationship between depressed mood and alcohol use, however, remains complex. Although some research suggests that symptoms of depression are associated with alcohol use (G. Curran et al., unpublished data, 1999); Marlatt and Gordon, 1985; Schuckit and Montiero, 1988), others have found the relationship to be more complex (Turnbull and Gomberg, 1988; Vaglum, 1987) Again, research design and measurement issues may be implicated.

Very few studies have examined stress, coping, and depression as related to alcohol use by comparing crosssectional with situational reports. One exception, a study by Fromme and Rivet (1994), used both retrospective, trait measures and prospective reports of coping style to predict weekly alcohol use. Although significant correlations were found between reported typical alcohol use and actual reported alcohol use over a 10 day period, modest associations were found between reported typical coping and daily monitoring of coping style. The researchers, however, did not examine the effect of current depression on these relationships.

To address possible limitations of cross-sectional studies as well as various ways of measuring depression, I examined the relationship of depression (both self-reported depressive disorder and daily depressed mood) to use of alcohol to cope with stress in college students. I compared crosssectional measures of alcohol coping and depression with 45 consecutive daily measurements of the variables in an attempt to examine the relationship between depressed mood and use of alcohol coping. Specific aims of the study were (1) to examine the relationship between crosssectional and daily reports of alcohol coping, and whether symptoms of depression affect this relationship, (2) to examine the relationship between daily reported symptoms of depression and daily alcohol coping, and (3) to determine whether symptoms of depression affect the variability of alcohol coping over time.

\section{METHODS}

\section{Participants}

The study sample was drawn from a pool of male and female college students from upper level psychology courses. Students from these classes were given the opportunity to participate and to receive extra class credit for adequate completion of the study. Two hundred and fifty-three students volunteered initially. At the completion of the study, 127 participants ( $78 \%$ female, $22 \%$ male) had met inclusion criteria (turned in at least 45 of the 50 data sheets no more than 7 data sheets at one time at any point in the study) and were included in the final analyses. Subjects ranged in age from 19 to 42 (mean age 20.5, SD 4.2). I maintained confidentiality by using only the last six digits of a participant's social security number in conjunction with the data. Chi-square analyses revealed no significant differences in depression group status (i.e., currently depressed, never depressed, or formerly depressed) or in use of trait alcohol coping between those who completed the study and those who did not.

\section{Procedure}

Participants initially were given a battery of mcasures to complete at home and return within 1 week. This packet consisted of a consent form, the Beck Depression Inventory (BDI; Beck, 1974), the Inventory to Diagnose Depression (IDD)-Lifetime version (IDD-L), the IDD-Past Week version (IDD-PW; Zimmerman and Coryell, 1987), and the dispositional version of the COPE Inventory (Carver et al., 1989). Participants were then given a second packet consisting of 50 computer response sheets and a daily questionnaire that was to be completed and returned each day for 50 days. The participants were instructed to complete the questionnaire at the end of each day. At the end of the data collection phase, participants completed an anonymous survey concerning their level of compliance with the procedures. The majority of the participants $(78 \%)$ indicated that they completed most of their questionnaires at the end of the day as instructed, and $85 \%$ of the sample reported that they responded mostly or completely honestly to the daily questionnaire items.

\section{Measures}

Case Identification. The IDD (Zimmerman and Coryell, 1987) was used to assess depression in this study. The IDD-L assesses the weck in the subject's life in which he or she felt the most depressed. The IDD-PW assesses depressive symptoms in the present week of the participant's life. The IDD can identify participants with major depressive disorder (MDD) based on DSM-III-R criteria and is correlated with the other self-report scales; for example, $r=0.87$ for the BDI, and $r=0.80$ for the Hamilton Rating Scale. Reliability for this measure was also found to be quite high (Spearman-Brown split half reliability $=0.91$; Chronbach's alpha $=0.92$ ). Concurrent validity with this measure is also acceptable, with an overall agreement rate with the Diagnostic Interview Schedule (Helzer and Robins, 1988). The IDD-PW includes symptoms that have been present over the previous week. This measure was scored dichotomously, classifying participants as either meeting DSM criteria for depression or not based on symptom endorsements. Participants who met diagnostic criteria for MDD on this inventory were classificd as currently depressed. Participants who were not deemed currently depressed by this measure, yet had been depressed in the past (again, using DSM diagnostic criteria for the presence of an episode of MDD) as measured by the IDD-L, comprised the formerly depressed group. The BDI-Short Form (BDI-SF) was used to ensure the absence of current depressive symptomatology.

\section{Diagnostic Group Classification}

Measures from the initial packet of questionnaires (including the IDD, IDD-L, BDI-SF, and COPE) were gathered and scored to classify participants as currently depressed (CD), formerly depressed (FD), or never depressed (ND). Individuals were classified as $C D$ if they met the criteria for depression on the IDD-PW. Formerly depressed individuals were so 
classified if they did not meet the criteria for depression on the IDD-PW but did meet depression criteria on the IDD-L. To verify a lack of current depressive symptomatology, the FD individuals also had to have scored in the subclinical range on the BDI-SF (a score <9). ND individuals scored in the nondepressed range on each of the depression measures (IDD, IDD-L, and BDI-SF). Of the participants who did not complete the study ( $n=126), 68 \%(n=87)$ were from the ND group, $24 \%(n=31)$ from the FD group, and $7 \%(n=9)$ from the CD group. With these criteria, the sample used in the final analyses consisted of $17 \mathrm{CD}$ participants, $38 \mathrm{FD}$ participants, and 72 ND participants.

Dispositional Coping. Dispositional alcohol coping style was assessed using the COPE Inventory (Carver et al., 1989). The COPE is a 52-item measure that was developed to measure a wide range of potential coping responses to stressors and to distinguish each one as well as possible from others. Each item is worded in the first person and indicates typical use of alcohol to cope with stress. The instructions of the dispositional version note that different stressful events bring out different patterns of responses, and respondents are asked to indicate how they usually react to stressors. Each item is rated on a 4-point scale with $1=I$ usually don't do this at all, $2=I$ usually do this a little bit, $3=I$ usually do this a medium amount, and $4=I$ usually do this a lot. Participants were asked to use this 4-point scale to report on the extent to which they typically responded to stressful events by using alcohol. Examples of alcohol coping items are "I try to lose myself for a while by using alcohol" and "I use alcohol to help me get through it." This measure has adequate test-retest reliability ( $r=$ 0.61 ).

Situational Alcohol Coping. The situational version of COPE (Carver et al., 1989) is modified to reflect the extent to which respondents used alcohol to cope with the most stressful event of each day for the 45 days of the study. This version used the same 4 point Likert-type scale as the dispositional COPE. Thus, each day, participants indicated the most stressful event of the day and then reported (on the 4-point scale) the extent to which they used alcohol to cope with this event. Seventy percent of the sample reported some days of using alcohol to cope with daily stress.

Daily Depressed Mood. Participants were asked to report their level of symptoms of depression and anxiety daily using the Dysphoria (five items) and Anxiety (three items) scales of the Daily Experiences Survey (J.E. Hokanson et al., unpublished data, 1994), which was developed to briefly assess a variety of constructs daily. Each scale was designed to tap distress associated with each symptom of depression and anxiety; the scales were derived from existing measures with adequate psychometric propertics and have high correlations with these scales $(0.79$ with Brief Symptom Inventory, Depression scale, Derogatis, 1983; and 0.73 with the State-Trait Anxiety Inventory, Spielberger, 1976, respectively).

Each mood item was also rated on a 5-point Likert-type scale, with 0 indicating that the participant did not feel that way at all and 4 indicating that the participant was extremely distressed by the symptom.

\section{RESULTS}

Before conducting analyses, I examined possible gender differences in dispositional alcohol coping, daily alcohol coping, and daily depression. No significant gender differences were found on any of these variables.

\section{Relationship Between Cross-Sectional and Daily Reports of Alcohol Coping}

To demonstrate the correspondence between an individual's report of the extent to which he or she typically used alcohol to cope and what the individual actually reported daily in response to specific daily stressors, I examined the relationship between daily and dispositional reports of coping. The effect of the presence of a depressive disorder on this relationship was also of interest. Each participant's scores on a dispositional alcohol coping scale (see Measures section) were compared to daily alcohol coping scores, which were obtained based on composite scores on the daily alcohol coping measures (i.e., average ratings on the measure summed across 50 days). Bivariate correlations between cross-sectional and daily reports of alcohol coping were examined separately for nondepressed and depressed participants. Significant correlations were obtained between cross-sectional and daily reports of alcohol coping $(r=0.60, p<0.01)$. However, for CD participants, the correlation between cross-sectional and daily reports of alcohol coping was not significant. When CD and FD participants were examined together, the strength of the significant correlation was greater $(r=0.66, p<0.01)$.

\section{Relationship Between Daily Reports of Alcohol Coping and Depression}

I examined the relationship between daily alcohol coping and depression using two different measures of depression: (1) a baseline diagnosis of MDD, and (2) each participant's average daily depressed mood score, which reflected a composite of each participant's daily score averaged across the 45 days of the study. Analyses performed using the daily depressed mood score for each participant revealed a significant relationship between mean daily alcohol coping and daily depressed mood $(r=0.28, p<0.01)$.

I also examined differences between CD and ND participants in daily reports of alcohol coping by using depression diagnoses as measured by the IDD-PW. Again, mean daily alcohol coping scores were used as the dependent variable. A one-way ANOVA revealed nonsignificant diagnostic group differences between ND and CD participants in daily use of alcohol to cope. Nonsignificant differences were also found when the FD group was included in the analysis.

\section{Relationship Between Depression and Variability of Alcohol Coping}

To determine whether symptoms of depression affect the variability of alcohol coping over time, I examined depression diagnostic group differences as well as high and low depression mood groups. These groups were constructed based on a median split of depressed mood symptoms over the 45 days of the study. Participants in the high daily depressed mood group showed significantly more variability in the daily use of alcohol coping (Mann-Whitney $U$ test $=480, p<0.01)$. However, analysis of diagnostic group differences (based on time 1 diagnosis of depression) revealed no significant group differences in the variability of alcohol coping.

\section{DISCUSSION}

In the present study I aimed to compare the crosssectional and daily reports of alcohol coping in college 
students and determine whether self-reported depression affected this relationship. I also examined the relationship between daily reported depressed mood and daily alcohol coping.

Results show that the relationship between crosssectional reports and across-time reports of using alcohol to cope with stress differs depending on whether an individual is experiencing symptoms of depression. In this sample, the correspondence between reports of typical alcohol use to cope with stress and actual daily reported use was influenced by the presence of self-reported depression. Among the depressed group, there was no significant correlation between dispositional and daily reports of alcohol coping. Studies that pool depressed and nondepressed participants may, however, find a relationship between the two methods of reporting (as was the case in this study when the groups were examined together; see also Fromme and Rivet, 1994). Therefore, the presence of depression may lead to inaccurate reporting of alcohol use to cope as measured cross-sectionally. Moreover, aaily reports of depressed mood are related to daily reports of use of alcohol to cope, however, no differences in daily alcohol coping were found between depressed and never depressed diagnostic groups. Therefore, the use of alcohol to cope with stress may be related to daily symptoms of depression and may not necessarily be related to the presence of an episode of depression (as measured by a diagnostic instrument). This finding points to the utility of assessing symptoms of depression when implementing and evaluating programs designed to improve coping skills of college students, including relapse prevention strategies that incorporate coping skills training. This recommendation is supported by findings which suggest that post-alcohol-treatment depression or depressive symptomatology is related to worse drinking outcomes (G. Curran et al., unpublished data, 1999).

Conversely, the presence of a depressive disorder may be unrelated to the likelihood that a college student will use alcohol in response to stress. In this study I did not employ measures of quantity and frequency of alcohol use or of alcohol-related consequences. Therefore, no conclusions about the relationship of alcohol-related problems to depression in college students can be drawn from these findings. However, previous studies indicate that use of alcohol in response to stress may be related to more frequent drinking (Hittner, 1995) and may continue to increase in postcollegiate years (Perkins, 1999).

Daily collection of study variables also allowed me to examine the variability of alcohol use in response to stress over time. Those who reported daily depressed mood showed more variability in use of alcohol to cope than did those with no symptoms of depression. However, no differences were found in variability of alcohol coping between depressed and never depressed diagnostic groups. Again, the relationship between depression and alcohol coping is different depending on how (diagnostic status versus symptom count) and when (cross-sectionally or longitudinally) depression is measured. It seems that depressed college students as a group in this sample (diagnosed at baseline) tended to not report use of alcohol to cope; therefore, variability would not be expected. It can be speculated that the relationship of variable alcohol coping among those with depressed daily mood may reflect a causal relationship between these two variables. For example, depressed mood may itself constitute an unpleasant mood state that a student may expect alcohol use will alleviate. Alternatively, use of alcohol has been found to contribute to depressed mood, even at low doses (Hesselbrock and Hesselbrock, 1993). Future studies that include valid measures of alcohol use and depressed mood over time are needed to elucidate any causal relationships more specifically.

Previous studies have suggested the potential importance of gender differences in alcohol use and coping in college students. The results of this study did not indicate any gender differences in any of the analyses conducted. This is not surprising, because gender differences are more likely to be found when quantity and frequency of alcohol use are measured rather than coping style (Fromme and Rivet, 1994). Also, in studying stress-motivated drinking in college students, Perkins (1999) found similar patterns of alcohol use in response to stress for males and females.

Overall implications for research design indicate that assessment of alcohol use in response to stress at one point in time may not be a valid indicator across time and situations. These findings suggest that symptoms of depression may be associated with inaccurate reporting of alcohol use in cross-sectional research designs. In addition, studies that assess more severe symptoms of depression in relation to alcohol use may ignore influences of less severe mood symptoms.

This study was intended to examine the relationships of different measurements of self-reported depression to reports of alcohol use in response to stress. Therefore, direct reports of quantity and frequency of alcohol use and diagnostic measures of alcohol use disorders were not employed. Future studies should focus on a broader spectrum of alcohol use and alcohol use disorders in community or treatment samples. Such studies will expand our understanding of the intricate relationships among various levels of alcohol and depressive disorders. Using a daily data collection design in comparison to a cross-sectional design revealed different relationships between these variables, which suggests that measurements taken at one point in time may indeed obscure results. Findings presented here support the utility of repeated measurement of depression and alcohol use in drawing conclusions about comorbidity.

\section{ACKNOWLEDGMENT}

I would like to acknowledge the support and assistance of my mentor, Dr. Jack E. Hokanson. 


\section{REFERENCES}

Beck AT, Beck RW (1974) Screening depressed patients in family practice: A rapid technique. Postgrad Med 52:8.

Brown SA, Schuckit MA (1988) Changes in depression among abstinent alcoholics. J Stud Alcohol 49:412-417.

Carver CS, Scheier MF, Weintraub JK (1989) Assessing coping strategies: A theoretically based approach. J Pers Soc Psychol 56:267-283.

Derogatis LR (1983) Brief Symptom Inventory. Clinical Psychometric Research, Baltimore.

Fromme K, Rivet K (1994) Young adults' coping style as a predictor of their alcohol use and response to daily event. I Youth Adolesc 23:85-97.

Helzer JE, Robins LN (1988) The Diagnostic Interview Schedule: Its development, evolution and use. Soc Psychiatry Psychiatr Epidemiol 23:6-16.

Hesselbrock MN, Hesselbrock VM (1993) Depression and antisocial personality disorder in alcoholism: Gender comparison, in Women and Substance Abuse (Gomberg ESL, Nirenberg TD eds), Ablex Publishing, Norwood, NJ.

Hittner JB (1995) Tension reduction expectancies and alcoholic beverages revisited: Associations to drinking frequency and gender. Int J Addiction 30:323-336.

Johnston LD, O'Malley PM, Bachman JG (eds) (1997) National survey results on drug use from the Monitoring the Future Study, 1975-1995: Vol. 2. College students and young adults (NIH Publication No. 94-3810). U.S. Government Printing Office, Washington DC.

Marlatt GA, Gordon JR (1985) Relapse Prevention: Maintenance Strategies in the Treatment of Addictive Behaviors. Guilford Press, New York.
Perkins HW (1999) Stress motivated drinking in collegiate and postcollegiate young adulthood: Life course and gender patterns. J Stud Alcohol 60:219-227.

Schuckit MA, Montiero MG (1988) Alcoholism, anxiety and depression. Br J Addict 83:1373-1380.

Spielberger CD (1976) The measurement of state and trait anxiety: Conceptual and methodological issues, in Emotions: Their Parameters and Measurement (Levi L ed), pp. 713 725, Raven Press, New York.

Stader SR, Hokanson JE (1998) Psychosocial antecedents of depressive symptoms: An evaluation using daily experiences methodology. J Abnorm Psychol 107:17-26.

Stone AA, Lennox S, Neale JM (1985) Daily coping and alcohol use in a sample of community adults, in Coping and Substance Use (Shiffman S, Wills TA eds), pp 199-220, Academic Press, Orlando, FL

Turnbull JE, Gomberg ESL (1988) Impact of depressive symptomatology on alcohol problems in women. Alcohol Clin Exp Res 12:374-381.

Vaglum S, Vaglum P, Larsen O (1987) Depression and alcohol consumption in non alcoholic and alcoholic women: A clinical study. Acta Psychiatr Scand 75:577-584.

Weinberger DA, Bartholomew K (1996) Social-emotional adjustment and patterns of alcohol use among young adults. J Pers 64:495-527.

Zimmerman M, Coryell W (1987) The Inventory to Diagnose Depression (IDD): A self report scale to diagnose major depressive disorder. J Consult Clin Psychol 55:55-59. 